González L., Martí E., Calvo I., Ruiz A., Pérez J. (2018) Towards Risk Estimation in Automated Vehicles Using Fuzzy Logic. In: Gallina B., Skavhaug A., Schoitsch E., Bitsch F. (eds) Computer Safety, Reliability, and Security. SAFECOMP 2018. Lecture Notes in Computer Science, vol 11094. Springer, Cham

The final publication is available at Springer via https://doi.org/10.1007/978-3-319-99229-7 24 


\title{
Towards Risk Estimation in Automated Vehicles Using Fuzzy Logic
}

\author{
Leonardo González ${ }^{1,2}$, Enrique Martí ${ }^{1}$, Isidro Calvo $^{2}$, Alejandra Ruiz ${ }^{1}$, \\ Joshue Pérez ${ }^{1}$ \\ ${ }^{1}$ Tecnalia Research and Innovation \\ leonardo.gonzalez, enrique.marti, alejandra.ruiz, \\ joshue.perez $\{$ @tecnalia.com\} \\ ${ }^{2}$ University of the Basque Country \\ isidro.calvo@ehu.edu
}

\begin{abstract}
As vehicles get increasingly automated, they need to properly evaluate different situations and assess threats at run-time. In this scenario automated vehicles should be able to evaluate risks regarding a dynamic environment in order to take proper decisions and modulate their driving behavior accordingly. In order to avoid collisions, in this work we propose a risk estimator based on fuzzy logic which accounts for risk indicators regarding (1) the state of the driver, (2) the behavior of other vehicles and (3) the weather conditions. A scenario with two vehicles in a car-following situation was analyzed, where the main concern is to avoid rear-end collisions. The goal of the presented approach is to effectively estimate critical states and properly assess risk, based on the indicators chosen.
\end{abstract}

Keywords: automated vehicles, collision avoidance, fuzzy logic, timeto-collision,driving behavior

\section{Introduction}

In a non-automated vehicle, risk in the environment is processed by the driver, who intuitively analyzes several factors in a typical driving scenario, such as the distance to the obstacles, the relative velocities to other vehicles, the roadway conditions, the traffic rules, among other environment and vehicle variables. Increasing vehicle automated capabilities requires their awareness of the environment to grow accordingly, so that they are able to understand and process different threat sources. A prompt and detection of dangerous situations, will allow vehicles to react and avoid or mitigate accidents.

Nowadays, traffic accidents are responsible for up to $1.25 \mathrm{M}$ deaths and $50 \mathrm{M}$ injuries worldwide annually according to the World Health Organization [17]. The main reason for most accidents in rural and urban roads is human error, accounting for 94 percent of crashes according to the National Highway Traffic Safety Administration[16] . One of the main reasons to move towards automated vehicle technology is to improve safety by reducing the driver interaction with 
the system (taking the driver out of the loop). This transition is currently ongoing. Several functionality levels for the automated vehicle have been defined depending on how much the driver presence is required. For example, the Society of Automotive Engineers (SAE) has defined six levels of automation, depending on the vehicle capability to perform driving tasks in different scenarios (modes) [15].

Vehicular technology available in the market is on the verge of level 3 or "conditional automation", which performs monitoring and driving under restrict circumstances, while level 4 technology is currently being tested by several companies. On the other hand, "full automation" (level 5) describes a vehicle with full capability in all environments under dynamic circumstances, taking the driver out of the equation.

Automated vehicle technology is increasingly gaining attention in the industry, Advanced Driver Assistance Systems (ADAS) have been under research for several years. ADAS already provide level 2 automation in commercial vehicles, and paved the path to level 3 automation. Nonetheless, the reliability of these vehicles is limited, and still depends on the driver to take corrective actions if something goes wrong. The trend towards highly automated vehicles, nonreliant on driver intervention, makes vehicles fully responsible for all the driving actions. Thus, serious liability issues for vehicle manufacturers arise if safety is not thoroughly guaranteed.

This work develops a concept for online risk estimation, relying on safety properties in a conditionally automated vehicle (level 3), to provide a comprehensive global knowledge of the state situation in the driving action. This risk estimation will be studied for a rear end collision scenario, using a fuzzy logic approach.

The outline of this paper is as follows. First, a background of risk in automated vehicles is presented. Then, a general risk assessment architecture is proposed in section 2. In section 3 indicators of risk are selected and a fuzzy logic approach is described. In section 4, a case study is proposed to test our risk estimation. Finally conclusions are presented in sections 5 .

\section{Risk in Automated Vehicles}

Risk is related to a multitude of scenarios where the likelihood of loss or liability exists. For example, investment risk or project completion risk are associated with performance and value. In a general sense, Levenson [12] defines risk as a compound likelihood of components which may lead to a negative outcome.

The idea of risk in the automotive industry is associated with a vehicle incurring in a dangerous maneuver or scenario; a dangerous scenario is one that increases the likelihood of an automotive accident. According to Lefèvre [11], risk in automated vehicles is associated with the "likelihood and severity of the damage that a vehicle of interest may suffer in the future". In order to evaluate and minimize risks, it is necessary to have accurate mathematical models of the situation in order to predict threats. 
Most of the work regarding risk and threat assessment in the ITS (Intelligent Transportation System) community has been focused on trajectory prediction in order to detect collision states, which incurs huge computational cost [7]. The scenarios for collision avoidance vary greatly depending in the models used for forecasting. According to [11], these strategies can be categorized as future trajectory collision, binary collisions and probabilistic collision prediction. Some indicators have been proposed to obtain a direct measure of the risk, such as Time to collision (TTC), Time to lane change (TLC) mainly for lateral maneuvers, and Time to react (TTR) directly linked to the driver ability to take action under a threat.

More recently, risk has been associated with a set of "unusual events" as Lefevre argues in [10]; i.e. detecting a deviation from a standard behavior for a particular scenario. This concept of risk, based on an unexpected behavior, follows the principle that when a system is unable to predict the future actions of other participants, such actions may be regarded as risky or dangerous in a specific set of traffic rules. As a matter of example, a vehicle passing a red light is perceived as an increased risk for other vehicles in the scenario, given that such maneuver is not expected.

Detecting these unusual events requires the definition of a nominal behavior, which can be based on a series of rules. In [18] a context based risk detector was developed from a set of safety rules aimed at preventing adverse weather conditions and driver fatigue. In [14] Perez proposed an arbitration control which evaluates some risk-related indicators in order to negotiate a level of authority between the driver and system. This assessment is based on driver specific indicators depending on the attention and drowsiness, as well as TTC. These strategies aim at having a richer risk evaluation based not only on the physical model of the vehicle, but also on the environmental and driver situation.

\section{Risk Estimator}

Dynamic threats, presented during run-time behavior, represent a challenge for perception and decision systems. As a matter of example, the need for further research in risk assessment strategies has come up lately in the literature of automated driving as a mean to improve safety and provide reliability of its systems.

The architecture for automated vehicles used in this work is shown in figure 1, and is based on [9] where Lattarulo et al defined a framework to test controllers in a simulated scenario. Our approach makes use of the decision, perception and communication blocks. According to the architecture, the risk estimation component takes place before the behavioral planner, as this is the main subsystem responsible for action in the case of a high risk scenario. Also, our estimator requires information from the world in order to make the assessment as well as data from the perception component.

The perception component gets data from exteroceptive and proprioceptive sensors. Thus, it provides the vehicle with a situation awareness regarding its 


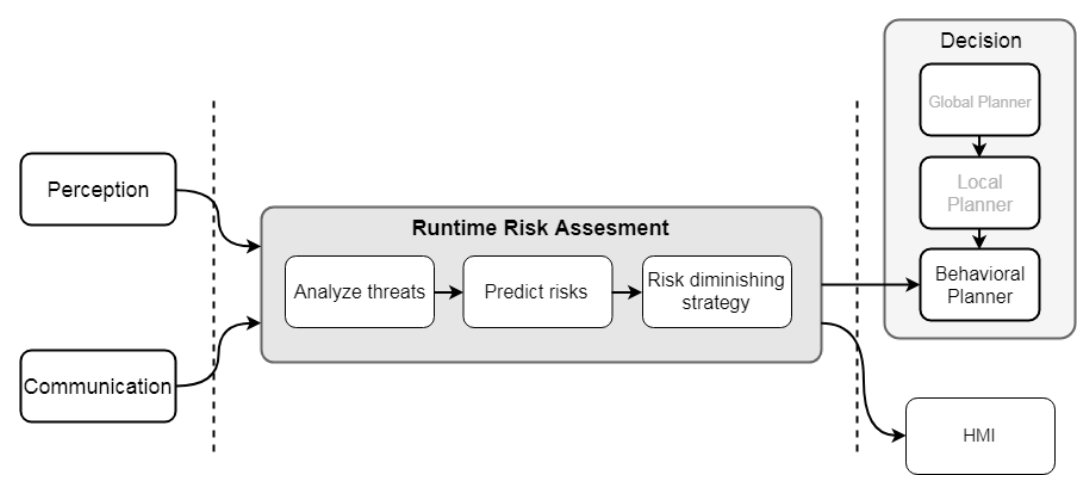

Fig. 1. Risk assessment architecture

environment and its current state. Accuracy on this environmental description depends heavily on the amount and type of sensors used. The environmental description includes, but it is not limited to, defining and identifying other participants, classifying them (e.g. vehicle, bus, cyclist, pedestrian), identifying road signals (pedestrian crossings, semaphores, etc. ), climatological conditions (fog, rain, snow and ice) among others.

The information of other vehicles states could also be provided by communication systems. Vehicle-to-vehicle communication (V2V) can greatly improve safety for automated and non-automated vehicles. The NHTSA predicts implementing V2V technology will diminish accident rates and greatly increased safety in the road [4].

Our Runtime Risk Assessment framework is composed of three main tasks: a) to recognize and properly assess threats, b) to predict risk situations regarding previously identified threats and, finally, c) to propose a risk-diminishing strategy; i.e. an action to avoid a risky situation. We consider a dangerous situation only regarding rear-end collisions in a single lane road. Based on this scenario three main variables were selected; time to collision (TTC), visual driver distraction (VDD) and weather conditions (WTHR). The scenario analyzed is composed of two vehicles in a car-following situation.

\subsection{Risk Estimation with Fuzzy Logic}

For the task of emulating human driving actions fuzzy logic control has been previously used in automated driving [13]. Since risk is inferred by the driver in a non-automated vehicle, a fuzzy logic approach was chosen to mimic this expertise. It allows extrapolating directly human knowledge in a set of rules to asses a situation's risk. For our system three main variables were selected, TTC, a weather representation, and visual driver distraction (VDD), representing the vehicle states, the driver and environment respectively as shown in figure 2 .

TTC is the main indicator for risk in our fuzzy logic approach, due to its direct relation with frontal collisions. This variable has been widely used in 


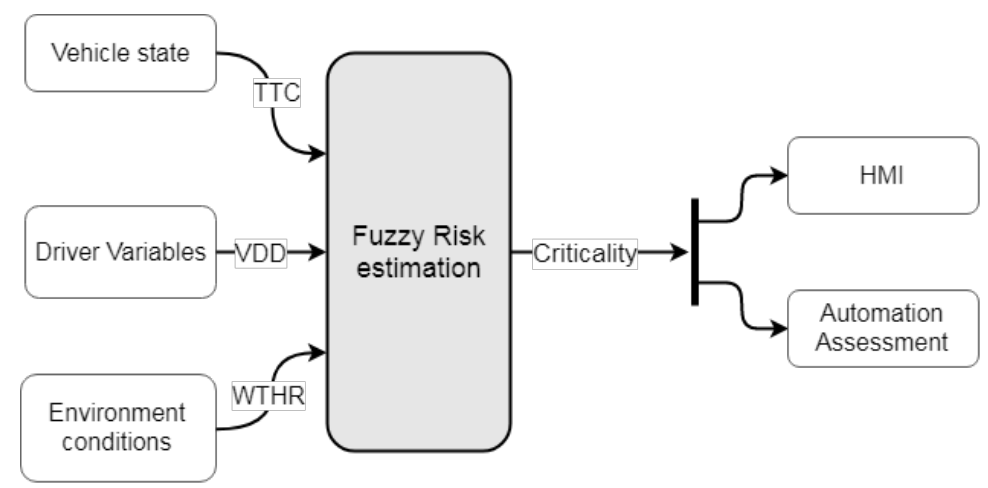

Fig. 2. Fuzzy System representation

the literature as an indicator of danger for vehicles. It was first introduced by Hayward [5] as an scalar measure of danger. TTC represents the time in the future in which a collision would have happened if the vehicle maintains its current speed and trajectory. This temporal indicator is critical if it is under 1.5 seconds [6] and it is generally consider safe when it is over 4 seconds. TTC is calculated using equation 1 , where $X_{i}$ is the position of $i$-th vehicle and $l_{i}$ its length.

$$
T T C=\frac{X_{i-1}(t)-X i(t)-l_{i}}{\dot{X}_{i-1}(t)-\dot{X} i(t)}
$$

Another input for the proposed fuzzy system is linked to the weather conditions. We used a normalized variable called WTHR. Weather affects the risk in a determined setup in multiple ways: it reduces the system (or driver) visibility and its ability to properly control the vehicle, it can also change the road conditions, diminishing the friction coefficient. In [8] a study to assess driver behavior and associate it with weather conditions was conducted, risk perception was associated with the ability of drivers to correctly assess the weather forecast.

Visibility and road condition, also change the behavior of other vehicles in a driving situation, so, a driver might behave more conservatively not only due to limited control, but also because other vehicles might exhibit the same limitations, making them hard to predict. Since these factors are complex to model for a vehicle, we used a simulated indicator, our WTHR indicator ranges from 0 to 1, where 0 represents optimal conditions for driving which could be translated to a dry roadway and good visibility.

Driver attention has also been included in the evaluation. Recently, efforts have been put into developing highly automated systems with drivers in the loop as a transitioning phase for autonomous technology, while still providing enhanced safety. One of the main challenges of such systems is being able to evaluate correctly when to intervene in a maneuver in order to take control of the vehicle from the driver. Other approaches manage the transition of authority between the automation and the driver dynamically [2]. These strategies 
are called sharing control, and the process of assessing an automation level has been labeled as arbitration control [3]. Our risk variable definition fits into such framework and can be taken into account as an indicator for when to perform such transition in a risky environment. In [1] a method to assess driver attention based on an analysis of head position and orientation was conducted, a Visual Driver Distraction (VDD) estimation was generated .To represent driver attention in our study an abstraction over VDD was used, our VDD indicator ranges from $[0,1]$, where 0 represents an optimal attention level (no distraction).

Our fuzzy logic approach defines three classes for the membership functions of each input, as shown in figure 3. These membership functions define a set of values related to each class, i.e. TTC is consider low below 2.5 seconds, and its membership to this set increases until it tops at 1 second and reaches its maximum membership. Membership functions allow to define subsets which might be overlapping, and different functions might be used, in our case (figure 3), these are created with triangular and trapezoidal functions. Fuzzification receives TTC, WTHR and VDD values and converts them to a fuzzy value using its membership functions. In our design we used a Mandani's fuzzy inference method, which allows for an if - then definition of our rules. The rule base provided in table 1, allows for a mapping of our inputs to our fuzzy output, or risk estimation. These rules are base on human expertise and knowledge, and allow for a soft mapping between inputs and risk states, which through defuzzificatio process outputs a crisp value, in our case a centroid method was used. For our fuzzy output membership function, five labels were designed to establish levels of criticality; safe, low warning, warning, low critical and critical. Our fuzzy system can be inspected as a function with three variables and a single output, a surface plot for our risk estimation is presented in figure 8 .
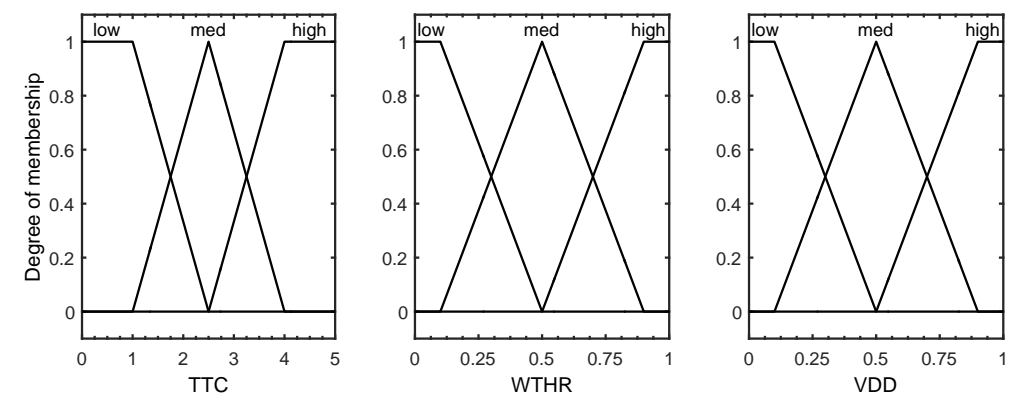

Fig. 3. Input membership functions for Fuzzy logic 

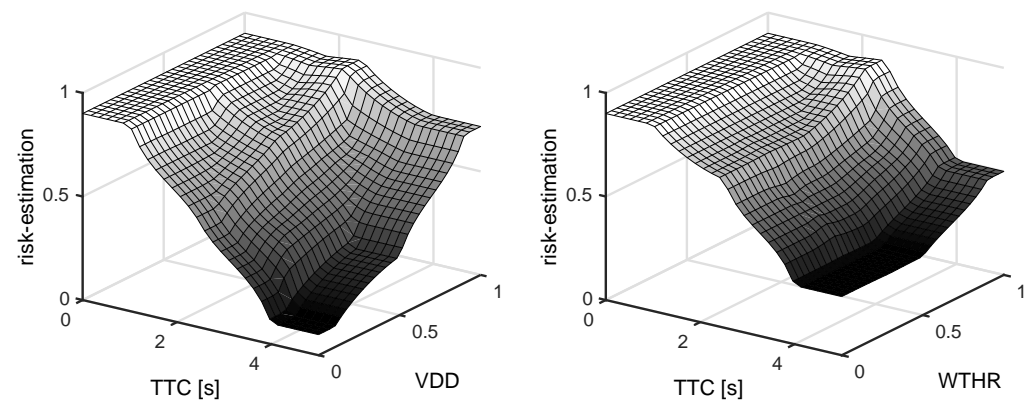

Fig. 4. Output surface functions

Table 1. Fuzzy system rule definition.

\begin{tabular}{|c|c|c|c|}
\hline TTC[s] & VDD & Weather & Fuzzy Output \\
\hline low & - & - & critical \\
med & low & low & low warning \\
med & med & low & low critical \\
med & high & low & critical \\
med & low & med & warning \\
med & med & med & low critical \\
med & high & med & critical \\
med & low & high & warning \\
med & med & high & critical \\
med & high & high & critical \\
high & low & low & safe \\
high & med & low & low warning \\
high & high & low & warning \\
high & low & med & low warning \\
high & med & med & low warning \\
high & high & med & warning \\
high & low & high & low warning \\
high & med & high & warning \\
high & high & high & warning \\
\hline
\end{tabular}

\section{Case Study}

Our study focuses on the analysis of rear-end collisions between two vehicles in a car-following scenario. The roadway used for our scenario was taken from a map, and intentionally contains a roundabout with an intersection in order to represent an actual urban street. This scenario is simple enough to test

the estimation made by our fuzzy logic approach. The main purpose of the test was to validate the risk estimation using different inputs for our indicators.

The simulation environment contains two vehicles, which communicate their states regarding velocity and position at all times. In a experimental scenario these states could be inferred from the sensors of the perception system, or simply shared through vehicle-to-vehicle $(\mathrm{V} 2 \mathrm{~V})$ communication. The trailing vehicle uses these states to compute the TTC, which is then fed to our fuzzy logic estimator along with our simulated variables.

TTC was calculated using equation 1 . The numerator represents the distance to collision (DTC). In our simulation this distance was assumed to be rectilinear 
regardless of the geometry of the road. This decision simplified calculations, and still portrays a worst case scenario, as this distance is always less or equal than the real distance on the road, yielding to a minimum TTC.

WTHR and VDD do not impact the simulation environment, instead they are only used by our risk estimation approach. In this way, they contribute indirectly to the TTC as main indicator, with driver and environment information, enhancing the estimation based on the set of rules established for our fuzzy logic approach.

For each vehicle a speed profile was also created, which depends on the geometry of the road and the maximum speed, acceleration and brake allowed. In this case, both maximum acceleration and brake were maintained equal among both vehicles, since our TTC calculation does not account for relative acceleration. Instead, different maximum speeds were imposed for each vehicle, making the leading vehicle slower to test the risk estimation in a impending collision. In order to observe how the risk estimation evolved, no modification was carried out by the system to avoid the collision, so the simulation continued until a collision effectively occurred. On the contrary, in a real situation the driver would avoid the collision by braking when he gets too close.

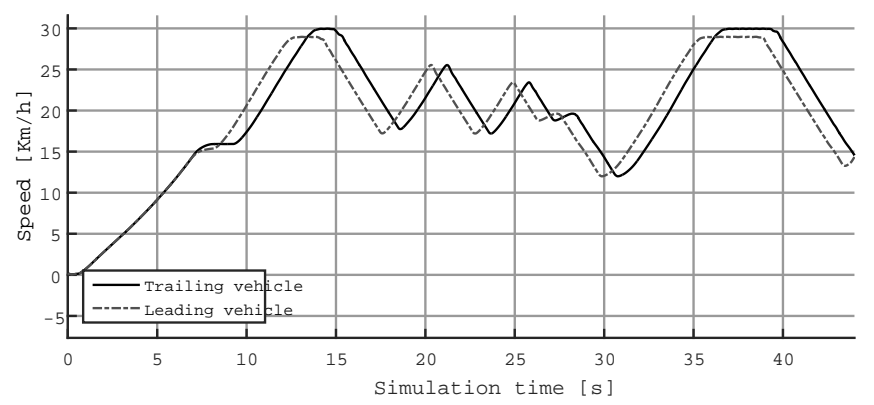

Fig. 5. Vehicles speed

\subsection{Results}

Simulations tested for 3 values of WTHR and 5 values of VDD. TTC was calculated during simulation time using the speed and position of both vehicles.

TTC for the trailing vehicle in figure 7 shows several peaks before collision. These peaks coincide with changes in curvature of the road, where vehicles diminish velocity and near miss situations occurred. This is observed five times before colliding with the vehicle in front at around 44 seconds according to the simulation.

In figure 8 all simulations are presented for different values of WTHR and VDD. Since WTHR is linked with weather conditions in the environment, it is expected to change less abruptly, though we are more concerned with grouping 


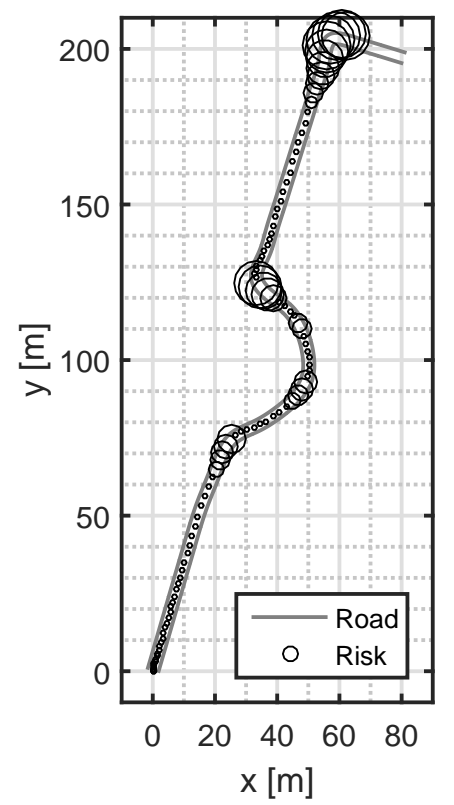

Fig. 6. Risk in road scenario. Circle size increases with higher risk.

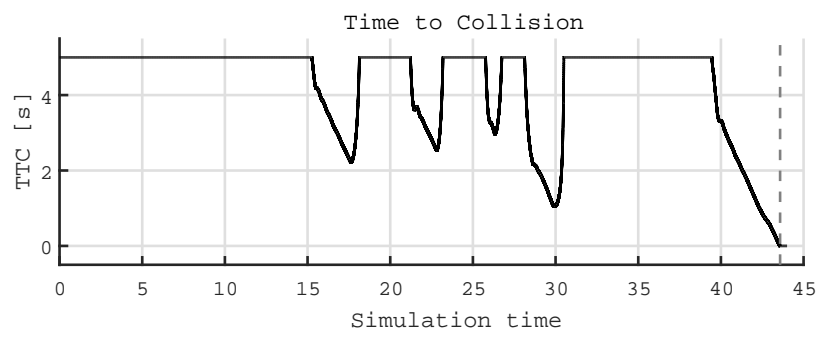

Fig. 7. TTC in our scenario 
VDD changes. Similar to TTC five peaks can be observed for all simulations, this was to be expected based on our rules.

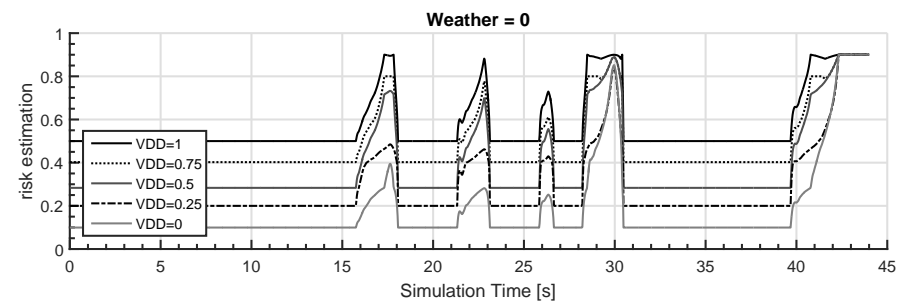

(a)

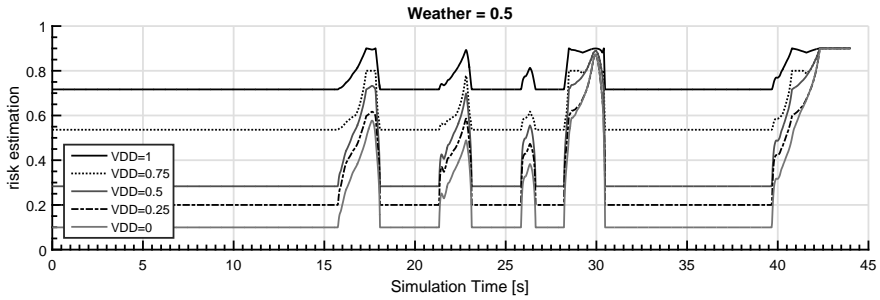

(b)

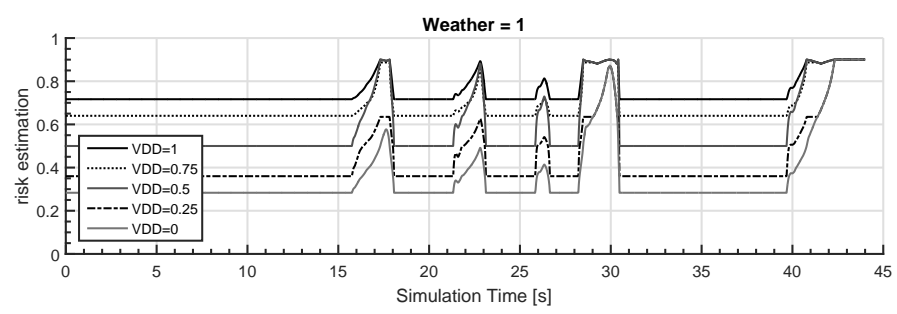

(c)

Fig. 8. Fuzzy output for different weather values

The first peak, after 15 seconds of simulation. coincides with the lead vehicle entering the roundabout, the change in curvature makes the longitudinal controller brake, reducing the distance between both vehicles and increasing relative speed. When the trailing vehicle brakes to also enter the roundabout our TTC calculation decrease quickly, leading to a nominal risk value. In Figure 6 this behavior of increasing risk close to curves, can be observed in the map. Two minor peaks can also be spotted inside the roundabout, both can be attributed to slight changes in curvature which modify the speed profile of the vehicle. Vehicles speed profile limits the maximum speed and acceleration of the vehicle as shown in figure 5 . 
The fourth peak observed corresponds to a near-miss scenario leaving the roundabout, in this case inter-vehicle distance got below one meter, and TTC dropped below 1.5 seconds. This situation resulted in a high estimation of risk before leaving the roundabout as can be seen in figure 6 . Finally our last peak corresponds to a collision, which occurs during the intersection at the end of our road scenario.

We can observe that geometry in the road had a direct impact over our estimation, due to the speed profile the vehicle used, even though it was not directly accounted in our estimation.

The emulated indicators helped to provide an enhanced risk estimation, by accounting for driver attention and road/weather conditions. In our results the WTHR indicator offsets our estimation without ever actually being the sole responsible for a high criticality. This complies with our initial design for which weather plays the role of making risky situations worse, by diminishing the controlability of the vehicle or the accuracy of sensors.

VDD has a more aggressive effect over our estimation. Again this is in check with the rules design for our fuzzy logic. Lower distraction levels correspond with safe states, where an increase in VDD quickly offsets our risk estimation.

\section{Conclusion}

Risk estimation in automated vehicles is a key issue in order to take proper decisions and adequate the driving behavior accordingly. This paper presents an exploratory approach for risk estimation based on fuzzy logic, since it allows embedding, in relatively easy way, the knowledge of human expertise into a simple set of rules. The outcome of the risk estimator allows inferring the level of risk taking into account (1) the state of the driver, (2) the behavior of other vehicle and (3) the weather conditions.

This approach was analyzed in a longitudinal collision scenario for a carfollowing maneuver in which two vehicles were involved. The rules designed provided a fast inference of risk, which allow the system to estimate critical levels when the prediction made by TTC was critical. In two events we observed a non-critical risk due to the curvature of the road, which affects our prediction by modifying the speed of the vehicles, these predictions were made critical when aggregated with sub-optimal weather conditions and driver attention.

In the future, more complex scenarios will be considered as well as different vehicle motion models. Assuming constant speed for TTC, limits the forecasting ability of the indicator. Road definition also needs to be leveraged in order to predict speed reductions when entering in curves with more precision, in order to expect low values of TTC in such circumstances.

Acknowledgments. This work was supported by the AMASS project (H2020ECSEL) with grant agreement number 692474. 


\section{References}

1. Boverie, S., Cour, M., Le Gall, J.Y.: Adapted human machine interaction concept for driver assistance systems driveasy. IFAC Proceedings Volumes 44(1), 2242-2247 (2011)

2. Flemisch, F., Heesen, M., Hesse, T., Kelsch, J., Schieben, A., Beller, J.: Towards a dynamic balance between humans and automation: authority, ability, responsibility and control in shared and cooperative control situations. Cognition, Technology \& Work 14(1), 3-18 (2012)

3. González, D., Pérez, J., Milanés, V., Nashashibi, F., Tort, M.S., Cuevas, A.: Arbitration and sharing control strategies in the driving process. Towards a Common Software/Hardware Methodology for Future Advanced Driver Assistance Systems p. 201 (2017)

4. Harding, J., Powell, G., Yoon, R., Fikentscher, J., Doyle, C., Sade, D., Lukuc, M., Simons, J., Wang, J.: Vehicle-to-vehicle communications: Readiness of v2v technology for application. Tech. Rep. DOT HS 812 014, National Highway Traffic Safety Administration, Washington, DC (August 2014)

5. Hayward, J.C.: Near miss determination through use of a scale of danger. Tech. rep., Pennsylvania Transportation and Traffic Safety Center (1972)

6. Van der Horst, A.R.A.: A time based analysis of road user behaviour in normal and critical encounters. No. HS-041 255 (1990)

7. Katrakazas, C., Quddus, M., Chen, W.H., Deka, L.: Real-time motion planning methods for autonomous on-road driving: State-of-the-art and future research directions. Transportation Research Part C: Emerging Technologies 60, 416-442 (2015), http://www.sciencedirect.com/science/article/pii/ S0968090X15003447

8. Kilpeläinen, M., Summala, H.: Effects of weather and weather forecasts on driver behaviour. Transportation research part F: traffic psychology and behaviour 10(4), 288-299 (2007)

9. Lattarulo, R., Pérez, J., Dendaluce, M.: A complete framework for developing and testing automated driving controllers. IFAC-PapersOnLine 50(1), 258-263 (2017)

10. Lefèvre, S., Laugier, C., Ibañez-Guzmán, J.: Evaluating risk at road intersections by detecting conflicting intentions. In: Intelligent Robots and Systems (IROS), 2012 IEEE/RSJ International Conference on. pp. 4841-4846. IEEE (2012)

11. Lefèvre, S., Vasquez, D., Laugier, C.: A survey on motion prediction and risk assessment for intelligent vehicles. ROBOMECH Journal 1(1), 1 (jul 2014)

12. Levenson, N.G.: System safety and computers. Addison Wesley (1995)

13. Llorca, D.F., Milanés, V., Alonso, I.P., Gavilán, M., Daza, I.G., Pérez, J., Sotelo, M.Á.: Autonomous pedestrian collision avoidance using a fuzzy steering controller. IEEE Transactions on Intelligent Transportation Systems 12(2), 390-401 (2011)

14. Pérez, J., González, D., Nashashibi, F., Dunand, G., Tango, F., Pallaro, N., Rolfsmeier, A.: Development and design of a platform for arbitration and sharing control applications. In: Embedded Computer Systems: Architectures, Modeling, and Simulation (SAMOS XIV), 2014 International Conference on. pp. 322-328. IEEE (2014)

15. SAE: Taxonomy and Definitions for Terms Related to On-Road Motor Vehicle Automated Driving Systems . Standard, Society of Automotive Engineers (Jan 2014)

16. Singh, S.: Critical reasons for crashes investigated in the national motor vehicle crash causation survey. Tech. rep., National Center for Statistics and Analysis (NCSA), NHTSA (February 2015) 
17. World Health Organization, WHO: Global status report on road safety 2015. Tech. rep., WHO (September 2015), [Online; accessed 11-September-2017]

18. Worrall, S., Orchansky, D., Masson, F., Nebot, E.: Improving vehicle safety using context based detection of risk. In: Intelligent Transportation Systems (ITSC), 2010 13th International IEEE Conference on. pp. 379-385. IEEE (2010) 